\title{
BORONIC ACIDS AS MECHANISTIC PROBES FOR THE BACTERIAL LUCIFERASE REACTION
}

\author{
Matthias Ahrens ${ }^{1}$, Peter Macheroux $^{1 *}$, Anatol Eberhard ${ }^{1} \dagger$, Sandro Ghisla $^{1} \ddagger$, \\ Bruce P. Branchaud ${ }^{2}$ and J. Woodland Hastings ${ }^{3}$ \\ 'Fakultät für Biologie, Universität Konstanz, W-7750 Konstanz, Germany, ${ }^{2}$ Department of \\ Chemistry, University of Oregon, Eugene, OR 97402, USA and ${ }^{3}$ The Biological Laboratories,
} Harvard University, Cambridge, MA 02138, USA

(Received 5 December 1990; accepted 4 February 1991)

\begin{abstract}
Bacterial luciferase uses long chain aldehydes as substrates. Alkylboronic acid analogs of these substrates with chain lengths of $C_{7}$ and $C_{9}$ have been synthesized, characterized, and used as mechanistic probes for the light emitting reaction. They behave as inhibitors in the in vitro luminescence reaction. Contrary to an earlier report (Macheroux and Ghisla, 1985, Nachr. Chem. Tech. Lab. 33, 785-790) they are not substrates for bacterial luciferase, in that they do not lead to light emission and are not oxidized by the flavin-4a-hydroperoxide to the products boric acid and the corresponding alcohol, as would be expected from a Baeyer-Villiger reaction. However, the particular conformation of a putative boronic acid hydroperoxide at the active center might be such that it would preclude a Baeyer-Villiger fragmentation. Thus, while the results do not support the postulate that luciferase proceeds via a Baeyer-Villiger mechanism, they also do not exclude it. A further observation was that the endogenous light emission (no added aldehyde) decays more rapidly than does the luciferase bound flavin-4a-hydroperoxide. This suggests that the endogenous light is not caused by the decomposition of the flavin-4a-hydroperoxide.
\end{abstract}

\section{INTRODUCTION}

Bacterial luciferase catalyzes the oxidation of reduced flavin mononucleotide $\left(\mathrm{FMNH}_{2}\right) \S$ and long chain aliphatic aldehydes by molecular oxygen to produce light, FMN, the corresponding carboxylic acid and water (Hastings et al., 1985). A long-lived luciferase-bound flavin-4a-hydroperoxide intermediate (FMNH-4a-OOH) is produced from the reaction of $\mathrm{FMNH}_{2}$ with $\mathrm{O}_{2}$ (Hastings and Gibson, 1963; Hastings et al., 1973), which then reacts with aldehyde to form a carboxylic acid and a luciferasebound flavin-4a-hydroxide in the singlet excited state (Kurfürst et al., 1984). The latter exhibits all the properties required for the emitter, and can be assumed to play this role at least in those cases in which no adventitious fluorescent protein emitters are involved in the reaction (Hastings et al., 1985; Ghisla et al., 1987; Eckstein et al., 1990).

The mechanism by which the aldehyde substrate is oxidized to the corresponding carboxylic acid (see below), thereby providing the energy for the generation of the excited state, is still elusive.

*Present address: University of Michigan, Department of Biological Chemistry, Ann Arbor, MI 48109, USA.

$\dagger$ Permanent address: Ithaca College, Department of Chemistry, Ithaca, NY 14850, USA.

$\ddagger$ To whom correspondence should be addressed.

$\S$ Abbreviations: FMN, flavin mononucleotide; FMNH-4a$\mathrm{OOH}, \mathrm{FMN}$-4a-hydroperoxide; $\mathrm{FMNH}_{2}$, reduced flavin mononucleotide; GC-MS, gas chromatography-mass spectroscopy; HPLC, high performance liquid chromatography; NMR, nuclear magnetic resonance.
An adaptation of the Baeyer-Villiger mechanism

$$
\begin{gathered}
\text { L-FMNH-4a-OOH }+ \text { R-CHO } \\
\rightarrow \text { L-FMNH-4a-OH }+ \text { R-COOH }+ \text { h } v
\end{gathered}
$$

( $c f$. Scheme 1) was proposed by Eberhard and Hastings (1972) to explain this process, and has been frequently quoted in textbooks and reviews as a viable path (Branchaud and Walsh, 1985). However, this mechanism suffers from the lack of a rationale for the generation of an excited state. It has been demonstrated that the flavoprotein cyclohexanone monooxygenase is able to process alkyland arylboronic acids as substrates, and good arguments have been put forward for this reaction proceeding via a Baeyer-Villiger type of mechanism (Branchaud and Walsh, 1985; Walsh and Chen, 1988). Furthermore, the oxidation of boronic acid substrate analogs to the corresponding boric acid
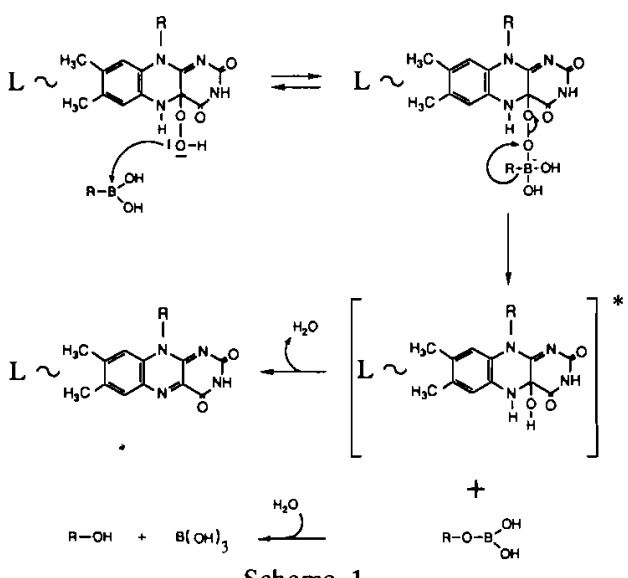

Scheme 1 
esters has been proposed to be diagnostic for this mechanism (Walsh and Chen, 1988). We thus attempted to verify a Baeyer-Villiger type of mechanism for the bacterial luciferase reaction by using the boronic acid analogs of the normal substrate, long chain aldehydes. Preliminary experiments (Macheroux and Ghisla, 1985) had indicated that such long chain boronic acids could yield light when substituted for aldehyde in the bacterial luciferase reaction. The interpretation of such results requires caution, however, because boronic acids are known to be very prone to oxidation, thus yielding aldehydes, the normal substrate of luciferase. We have carried out a thorough investigation of the reaction analogous to that depicted above, but substituting the corresponding boronic acid for aldehyde, and by taking care to analyze for all the potential products, i.e. light, boric acid, and nonanol. No light emission was observed and no evidence for the postulated products was obtained.

\section{MATERIALS AND METHODS}

1-Bromononane, trimethyl borate, and curcumin were from Merck (Darmstadt, Germany); FMN, decanal and boron standard solution were from Aldrich Chemical Co. (Milwaukee, WI). The aldehydes were distilled before use. All other chemicals were of reagent grade. All experiments were carried out in $10 \mathrm{mM}$ phosphate buffer, $\mathrm{pH} \mathrm{7,} \mathrm{con-}$ taining $0.35 \mathrm{M} \mathrm{NaCl}$ (standard buffer). Nonylboronic acid (CA Registry No. [3088-78-6]) and heptylboronic acid (CA Registry No. [28741-07-3]) were prepared according to published methods (Snyder et al., 1938; Torsell and Larsson, 1957). The crude material was stored under nitrogen at $-25^{\circ} \mathrm{C}$. Before use the material was crystallized once from nitromethane and twice from toluene. The white crystalline solid was moistened with water and stored in a brown jar under nitrogen at $-25^{\circ} \mathrm{C}$. Its ${ }^{1} \mathrm{H}-\mathrm{NMR}$ spectrum in deuterochloroform (JEOL, $90 \mathrm{MHz}$ ) was consistent with the structure and did not show any impurities.

Cells of Vibrio harveyi, Cline's mutant strain M-17, were grown and harvested as described by Nealson (1978). Luciferase was purified according to the procedure of Holzman and Baldwin (1982), and was $>95 \%$ pure as judged from SDS-gel electrophoresis. The concentration of the enzyme was estimated using a molecular weight of $79 \mathrm{kDa}$ and an $\mathrm{E}_{280}$ value of $74000 \mathrm{M}^{-1} \mathrm{~cm}^{-1}$ (Tu et al., 1977). Absorbance spectra were recorded with a Kontron Uvikon 820 spectrophotometer modified for the simultaneous determination of light emission, as described earlier (Kurfürst et al., 1984). The luciferase-bound FMNH4a-OOH was prepared and purified by molecular sieve column chromatography at $4^{\circ} \mathrm{C}$ (Kurfürst et al., 1984). After the decay of the FMNH-4a-OOH, the solution was stored at $-25^{\circ} \mathrm{C}$ until product analysis could be carried out. The content of boron was determined by using the curcumin method (Boltz and Gupta, 1970). Nonanol concentration was determined with the help of Dr. N. Blau (Department of Medicinal Chemistry, University of Zürich) by GC-MS, using a calibration curve extending over the range of 0.5 to $20 \mathrm{nmol}$. Bioluminescence was measured with a photometer as described by Mitchell and Hastings (1971). In a typical experiment, the reaction was initiated by the injection of $0.5 \mathrm{~mL}$ of an anaerobic solution containing $40 \mu M \mathrm{FMNH}_{2}$ (prepared by catalytic reduction of FMN with hydrogen over platinum) in standard buffer (see above) into a solution of luciferase (1 $\mu M)$ in standard buffer. Secondary additions were carried out immediately (1-2 s) using a syringe containing e.g. 0.5
$\mathrm{mL}$ of a saturated solution of nonylboronic acid in standard buffer. A concentration of $\sim \mathbf{4 0} \mu M$ was estimated for this solution by determining its boron content (Boltz and Gupta, 1970) upon oxidation for $30 \mathrm{~min}$ with $3 \% \mathrm{H}_{2} \mathrm{O}_{2}$ at pH 10 and $75^{\circ} \mathrm{C}$. In tertiary assays ( $c f$. Fig. 2) $0.5 \mathrm{~mL}$ of $0.6 \mu M$ decanal in standard buffer was injected followed by a solution of a boronic acid.

\section{RESULTS AND DISCUSSION}

\section{Preparation and purification of alkylboronic acids}

A most crucial point for this investigation is the purity of the boronic acids used. When nonylboronic acid was recrystallized from nitromethane as specified in the literature (Snyder et al., 1938; Torsell and Larsson, 1957) and used in the normal luciferase assay instead of decanal, light emission was indeed observed (Macheroux and Ghisla, 1985). However, the intensity depended on the age and mode of preparation of the sample. A careful evaluation of material which had been purified by various methods led to the conclusion that the occurrence of some light emission was due to the presence of a contaminant, most probably an aldehyde. For this study, nonylboronic acid was recrystallized from nitromethane and subsequently twice from toluene, collecting only the best fractions from each step. The boronic acid prepared by this method was homogeneous as judged by its ${ }^{1} \mathrm{H}-\mathrm{NMR}$ spectrum. Conventional chromatographic methods (such as HPLC) proved unsatisfactory as criteria for purity. It was stable at $-30^{\circ} \mathrm{C}$ in the presence of traces of moisture.

\section{Evidence for interaction of boronic acid with luciferase}

The luciferase bound flavin-4a-hydroperoxide (FMNH-4a-OOH) can be generated in situ by mixing luciferase, reduced flavin and oxygen. Its purification and characterization have been reported (Hastings et al., 1973; Becvar et al., 1978). The FMNH-4a-OOH reacts with long chain aldehydes to give rise to a blue-green light emission. Even without the addition of substrate a very low light emission occurs. This so-called endogenous light emission is typically less than $1 \%$ of the light emission obtained in the presence of an optimal aldehyde concentration (Hastings et al., 1966). The decay of the endogenous light emission is markedly biphasic with half lives of 3 and $13 \mathrm{~s}$ at $20^{\circ} \mathrm{C}$ (Fig. 1). Addition of nonylboronic acid to such in situ generated luciferase-bound FMNH-4a-OOH affects the course of the biphasic light emission, in that the first phase becomes significantly faster, while the second is essentially unaffected (half lives of 1 and 12 s, Fig. 1). Nonylboronic acid lowers the quantum yield by $30 \%$ as compared to a control. This demonstrates that nonylboronic acid binds to luciferase and affects the reactivity of the FMNH-4a-OOH. 


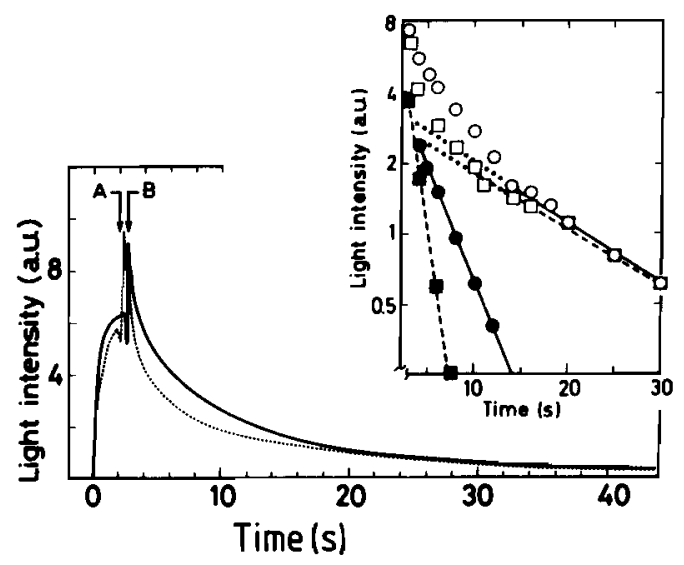

Figure 1. Effect of boronic acid on the luciferase endogenous light emission. The experiments were done by injecting at time $=0,0.5 \mathrm{~mL}$ of a $40 \mu M$ solution of $\mathrm{FMNH}_{2}$ into $1 \mu M$ luciferase in $1 \mathrm{~mL}$ standard buffer at $22^{\circ} \mathrm{C}$ in the absence of aldehyde. The solid curve shows the time course of light emission where, at the point in time (B) a secondary addition of $0.5 \mathrm{~mL}$ of standard buffer was made. The time course of light emission without the secondary addition was virtually identical with the solid curve with the exception of the "spike" denoted by the arrow (B) (data not shown). The dashed curve shows a similar experiment in which $0.5 \mathrm{~mL}$ of a $40 \mu M$ solution of nonylboronic acid was added secondarily at (A). The inset shows the semi-logarithmic plot of the same results in which circles and squares denote the emission in the absence and in the presence of boronic acid, respectively. The full symbols show the plot of the rapid part of the reaction obtained by subtracting the slow phase from the observed values. The $t_{1 / 2}$ for the slow phase in the absence of the boronic acid is $13 \mathrm{~s}$ while for the fast phase it is $3 \mathrm{~s}$. In the presence of the boronic acid, the respective $t_{1 / 2}$ values are 12 and $1 \mathrm{~s}$.

\section{Competition between aldehyde and boronic acid} for the active site

When nonylboronic acid was added to the reaction mixture in a normal assay subsequent to the addition of the aldehyde, the course of the aldehyde induced light emission was significantly modified: whereas the decay of the light intensity was monophasic with a half time of $6.5 \mathrm{~s}$ in the normal assay, it became distinctly biphasic in the presence of nonylboronic acid (Fig. 2) with half lives of 1 and $10 \mathrm{~s}$ at $20^{\circ} \mathrm{C}$. The effects of nonylboronic acid on the kinetics of light emission were very similar to those observed with long chain alcohols which are known to be competitive inhibitors (Hastings et al., 1966; Baumstark et al., 1979; Tu, 1979). Nonylboronic acid thus also competes with aldehyde for the active site of bacterial luciferase. Moreover the experiment described in Fig. 2 supports earlier observations that the interaction of the FMNH-4a-OOH with the aldehyde is reversible (Baumstark et al., 1979). It is interesting to note that the inhibitory effects of nonylboronic acid on the endogenous (Fig. 1) and aldehyde dependent (Fig. 2) light emissions are very similar.

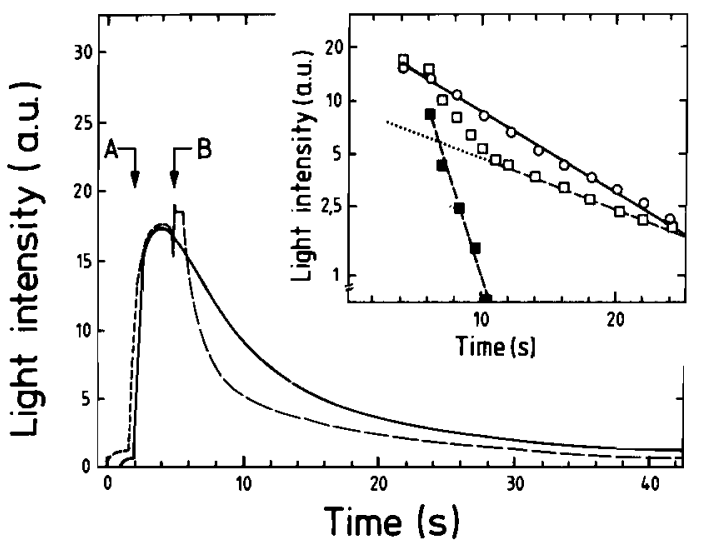

Figure 2. Effect of nonylboronic acid on the course of the luciferase reaction. The reaction conditions were similar to those described in the legend to Fig. 1. At time $=0$, FMNH ${ }_{2}$ and luciferase were mixed. At this point a low level of light emission ensued ("endogenous light"). After approximately $2 \mathrm{~s}, 0.5 \mathrm{~mL}$ of $0.6 \mu M$ decanal was added (A), which resulted in increase and subsequent decay of the light emission (solid curve). In a duplicate sample, 0.5 $\mathrm{mL}$ of a $40 \mu M$ nonylboronic acid solution was added at time point (B) (dashed curve). Addition of the same amount of buffer at (B) caused a "spike" of the light emission without changing the time course of the subsequent process (data not shown). The inset shows the semi-logarithmic plots of the light emission. Open circles and open squares denote the decay of the light emission in the absence and presence of boronic acid, respectively. The full squares show the fast phase observed in the presence of boronic acid: the values for the points were obtained by subtracting extrapolated values of the slow phase from the observed values.

When heptylboronic acid was added to a reaction mixture containing standard buffer, luciferase and decanal $(26 \mu M)$, and $\mathrm{FMNH}_{2}$ was subsequently injected, the maximum intensity of the light emitted was reduced compared to that obtained with the normal assay in the absence of the boronic acid (data not shown). Increasing amounts of heptylboronic acid gave decreasing amounts of light, with $50 \%$ inhibition at 50-100 $\mu M$ heptylboronic acid. This suggests that the $K_{\mathrm{i}}$ for heptylboronic acid is more than one order of magnitude higher than the $K_{\mathrm{d}}(2.4 \mu M)$ measured for the dissociation of the corresponding alcohol (octanol) from the luciferaseFMNH-4a-OOH intermediate (Tu, 1979).

\section{Reaction of nonylboronic acid with purified lucifer- ase bound FMNH-4a-OOH}

The experiments above have provided evidence that alkyboronic acids bind to bacterial luciferase and compete with the natural substrate for the active site, but do not themselves lead to light emission. We next addressed two questions. First, do alkylboronic acids stabilize the FMNH-4a-OOH, as was demonstrated earlier for long chain alcohols, and second, is it possible that alkylboronic acids become oxidized by the FMNH-4a-OOH in a dark reaction? 


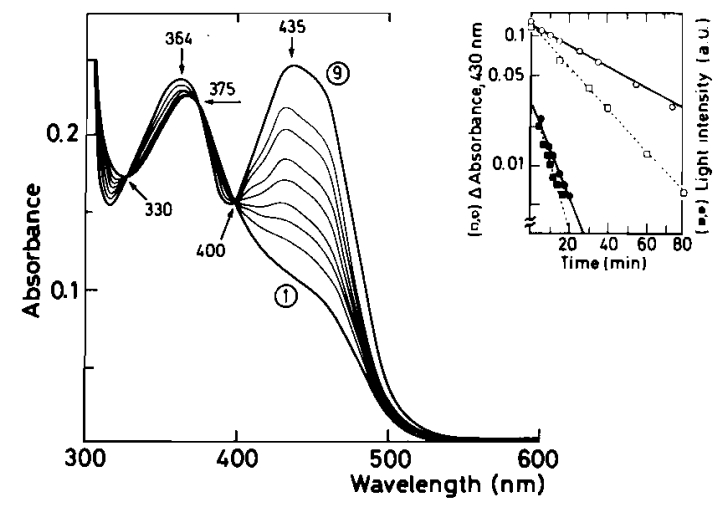

Figure 3. Effect of nonylboronic acid on the decay of luciferase-bound FMNH-4a-OOH and the decay of the endogenous light emission. The luciferase-bound FMNH4a-OOH was prepared and purified as described earlier (Kurfürst et al., 1984). The spectrum of $1 \mathrm{~mL}$ of this species in standard buffer at $4^{\circ} \mathrm{C}$ containing $24 \mu M$ boronic acid is shown by trace 1 . The subsequent curves were obtained after $5,10,15,25,35,55$, and $75 \mathrm{~min}$. The spectrum of curve 9 was obtained upon warming at ambient temperature for $5 \mathrm{~min}$ and subsequent recooling to $4^{\circ} \mathrm{C}$. The arrows indicate the absorbance maxima and the isosbestic points observed during the conversion. The initial spectrum and the changes observed with time were essentially identical in the absence of boronic acid (data not shown). The inset shows: absorbance decay in the absence $(O)$ and in the presence $(\square)$ of boronic acid; light emission intensity in the absence $(0)$ and in the presence ( $\boldsymbol{\square})$ of boronic acid. The light emission and absorbance spectra were measured with the same sample, using a spectrophotometer equipped to measure the two simultaneously (Kurfürst et al., 1984).

In order to answer these questions we monitored the decay of the purified FMNH-4a-OOH species in the absence and presence of highly purified nonylboronic acid (Fig. 3). The luciferase-bound FMNH$4 \mathrm{a}-\mathrm{OOH}$ was measured by its absorbance, and was found to decay more than twice as fast in the presence of nonylboronic acid $\left(t_{1 / 2}=17 \mathrm{~min}\right)$ than in its absence $\left(t_{1 / 2}=41 \mathrm{~min}\right)$. The decay of the endogenous light emission was only slightly faster in the presence of nonylboronic acid $\left(t_{1 / 2}=5.5\right.$ min compared to $7.5 \mathrm{~min}$ ). It has generally been assumed, and demonstrated to be so under some conditions (Hastings and Gibson, 1963), that the intensity of the endogenous emission is an accurate measure of the amount of luciferase-bound FMNH4a-OOH. Under the conditions used in the experiment of Fig. 3, however, the observed endogenous light emission had a much shorter half life than the decay of the FMNH-4a-OOH species. This observation indicates that the elusive endogenous light emission cannot be attributed to an excited state generated by the decay of the FMNH-4a-OOH itself. Finally, Fig. 3 shows that the decay of the FMNH-4a-OOH in the presence and absence of alkylboronic acids proceeds with the same isosbestic points. This finding implies that the nature of chemical events leading to the end product FMN are the same, even though the kinetics of the light emission and its quantum yield are different (Fig. 1).

Analysis of products from reaction of luciferase bound FMNH-4a-OOH with nonylboronic acid

Since the decay of the FMNH-4a-OOH is twice as fast in the presence of nonylboronic acid as in its absence, it is indeed conceivable that the nonylboronic acid is oxidized by the FMNH-4a-OOH, but that the process does not produce light. To clarify this point we have carried out a thorough search in the reaction mixture for the products to be expected from the peroxide oxidation of nonylboronic acid, i.e. 1-nonanol and boric acid (see Scheme 1). In an experiment such as that depicted in Fig. 3 and upon completion of the decay of the FMNH-4a-OOH to oxidized FMN, we did not detect any nonanol or boric acid. The sensitivity of the assay procedures (see Materials and Methods) would have allowed the detection of $0.7-2 \mathrm{nmol}$ of either product. Twelve nmol of luciferase-bound FMNH-4a-OOH were used in these experiments (cf. Fig. 3), so this means that less than $15 \%$ and more probably less than $5 \%$ of the decay of the luciferase-bound FMNH-4a-OOH could involve oxidation of the nonylboronic acid. From these results we conclude that alkylboronic acids do not react appreciably according to the reaction sequence depicted in Scheme 1. The accelerated decay of the FMNH-4a-OOH in the presence of alkylboronic acids thus can not be attributed to the oxidation of the boronic acid. Instead, binding of alkylboronic acid to luciferase may induce a conformational change of the protein which in turn destabilizes the FMNH-4a-OOH. If any oxidation of boronic acids occurs, it must be slow, and any putative light emission would have to be considerably less than the endogenous light emission. If it occurs at all, it should be considered as a side reaction. It is also possible that the boronic acids perturb the hydrogen bonding network of the enzyme intermediates or directly catalyze $\mathrm{H}_{2} \mathrm{O}_{2}$ elimination from the luciferase-FMNH-4a-OOH.

\section{CONCLUSION}

The results presented demonstrate that alkylboronic acids do not substitute for aldehydes as substrates for bacterial luciferase. Instead, they are inhibitors of the normal luciferase reaction. These results are surprising since boronic acids are very easily oxidized by flavin hydroperoxides (Branchaud and Walsh, 1985) and by two flavoprotein monooxygenases which have been presumed to proceed through the same FMNH-4a-OOH intermediate found in bacterial luciferase. It is conceivable that stereoelectronic factors could be responsible for the lack of reaction of boronic acids with the luciferasebound FMNH-4a-OOH. Baeyer-Villiger reactions 


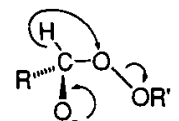

A1

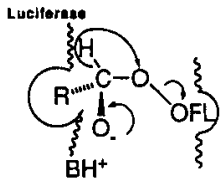

A2

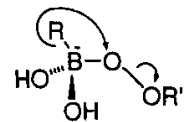

B1

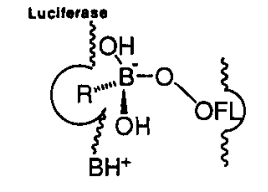

B2

Scheme 2

and boronic acid oxidations are generally assumed to proceed in an antiperiplanar configuration, as shown in Scheme 2, structures A1 and B1, respectively, in order to maintain optimal orbital overlap as the carbon-oxygen bond forms and the oxygenoxygen bond cleaves. The tetrahedral adduct is probably bound to the luciferase by the alkyl side chain of the aldehyde, by the flavin and perhaps by an acid-base catalytic group in the conformation shown in Scheme 2, structure A2, optimally aligned for hydride migration. If alkylboronic acids are bound in a similar fashion then alkyl migration cannot proceed because the alkyl group is bound by the enzyme and thus the carbon-boron bond is not properly oriented antiperiplanar to the oxygenoxygen bond (Scheme 2, structure B2).

Acknowledgements-This work was supported in part by grants from the Deutsche Forschungsgemeinschaft to S.G. (Gh 2/4-6), and the National Science Foundation to B.P.B. (DMB 87-17730) and to J.W.H. (DMB 86-16522). Part of the work was carried out while A.E. was on a sabbatical leave in Konstanz from Ithaca College.

\section{REFERENCES}

Baumstark, A. L., T. W. Cline and J. W. Hastings (1979) Reversible steps in the reaction of aldehydes with bacterial luciferase intermediates. Archs Biochem. Biophys. 193, 449-455.

Becvar, J. E., S.-C. Tu and J. W. Hastings (1978) Activity and stability of the luciferase-flavin intermediate. Biochemistry 17, 1807-1812.

Boltz, D. F. and H. K. L. Gupta (1978) Boron. In Chemical Analysis, Vol. 8, Colorimetric Determination of Nonmetals (Edited by D. F. Boltz and J. A. Howell), pp. 1-37. Wiley, New York.

Branchaud, B. P. and C. T. Walsh (1985) Functional group diversity in enzymatic oxygenation reactions catalyzed by bacterial flavin-containing cyclohexanone oxygenase. J. Am. Chem. Soc. 107, 2153-2161.

Eberhard, A. and J. W. Hastings (1972) A postulated mechanism for the bioluminescent oxidation of reduced

flavin mononucleotide. Biochem. Biophys. Res. Commun. 47, 348-353.

Eckstein, W. J., K. W. Cho, P. Colepicolo, S. Ghisla, J. W. Hastings and T. Wilson (1990) A time-dependent bacterial bioluminescence emission spectrum in an in vitro single turnover system: Energy transfer alone cannot account for the yellow emission of Vibrio fischeri Y-1. Proc. Natl. Acad. Sci. USA 87, 1466-1470.

Ghisla, S., J. Eckstein and P. Macheroux (1987) On the chemical mechanism of bacterial luciferase, an updating. In Flavins and Flavoproteins (Edited by D. E. Edmondson and D. B. McCormick), pp. 601-612. Walter deGruyter, New York.

Hastings, J. W., C. Balny, C. LePeuch and P. Douzou (1973) Spectral properties of an oxygenated luciferase-flavin intermediate isolated by low-temperature chromatography. Proc. Natl. Acad. Sci. USA 70, 3468-3472.

Hastings, J. W. and Q. H. Gibson (1963) Intermediates in the bioluminescent oxidation of reduced flavin mononucleotide. J. Biol. Chem. 238, 2537-2554.

Hastings, J. W., Q. H. Gibson, J. Friedland and J. Spudich (1966) Molecular mechanisms in bacterial bioluminescence: on energy storage intermediates and the role of aldehyde in the reaction. In Bioluminescence in Progress (Edited by F. H. Johnson and Y. Haneda), pp. 151-186. Princeton University Press, NJ.

Hastings, J. W., C. J. Potrikus, S. Gupta, M. Kurfürst and J. C. Makemson (1985) Biochemistry and physiology of bioluminescent bacteria. Adv. Microb. Physiol. 26, 235-291.

Holzman, T. F. and T. O. Baldwin (1982) Isolation of bacterial luciferases by affinity chromatography on 2,2 . diphenylpropylamine-Sepharose: phosphate-mediated binding to an immobilized substrate analogue. Biochemistry 21, 6194-6201.

Kurfürst, M., S. Ghisla and J. W. Hastings (1984) Characterization and postulated structure of the primary emitter in the bacterial luciferase reaction. Proc. Natl. Acad. Sci. USA 81, 2990-2994.

Macheroux, P. and S. Ghisla (1985) Die bakterielle Biolumineszenz. Nachr. Chem. Tech. Lab. 33, 785-790.

Mitchell, G. W. and J. W. Hastings (1971) A stable, inexpensive, solid state photomultiplier photometer. Anal. Biochem. 39, 243-250.

Nealson, K. H. (1978) Isolation, identification, and manipulation of luminous bacteria. In Methods Enzymol. (Edited by M. A. DeLuca), Vol. 57, pp. 153-166. Academic Press, New York.

Snyder, H. R., J. A. Kuck and J. R. Johnson (1938) Organoboron compounds, and the study of reaction mechanisms. Primary aliphatic boronic acids. J. Am. Chem. Soc. 60, 105-111

Torsel1, K., and E. N. V. Larsson (1957) Darstellung langkettiger Alkylborsäuren. Acta Chem. Scand. 11, 404-405.

Tu, S.-C. (1979) Isolation and properties of bacterial luciferase-oxygenated flavin intermediate complexed with long-chain alcohols. Biochemistry 18, 5940-5945.

Tu, S.-C., T. O. Baldwin, J. E. Becvar and J. W. Hastings (1977) Bacterial luciferase activity does not require a disulfide-dithiol conversion. Arch. Biochem. Biophys. 179, 342-348.

Walsh, C. T. and Y.-C. J. Chen (1988) Enzymic Baeyer-Villiger oxidations by flavin-dependent monooxygenases. Angew. Chem. Int. Ed. Engl. 27, 333-343. 